\title{
$S U(2)$-spin Invariant Auxiliary Field Quantum Monte-Carlo Algorithm for Hubbard models
}

\author{
F.F. Assaad \\ Institut für Theoretische Physik III, \\ Universität Stuttgart, Pfaffenwaldring 57, D-70550 Stuttgart, Germany.
}

\begin{abstract}
Auxiliary field quantum Monte Carlo methods for Hubbard models are generally based on a Hubbard-Stratonovitch transformation where the field couples to the $z$-component of the spin. This transformation breaks $S U(2)$ spin invariance. The symmetry is restored only after summation over the auxiliary fields. Here, we analyze an alternative decomposition, which conserves $S U(2)$ spin invariance, but requires the use of complex numbers. We show that this algorithm gets rid of the very large fluctuations observed in imaginary time displaced correlation functions of quantities which do not commute with the $z$-component of the total spin. The algorithm prooves to be efficient for the study of spin dynamics.
\end{abstract}

Auxiliary field quantum Monte Carlo Algorithms for Hubbard models are usually based on the discrete HubbardStratonovtich decomposition [1]:

$$
\exp \left(-\Delta \tau U \sum_{\vec{i}}\left(n_{\vec{i}, \uparrow}-\frac{1}{2}\right)\left(n_{\vec{i}, \downarrow}-\frac{1}{2}\right)\right)=\tilde{C} \sum_{s_{1}, \ldots, s_{N}= \pm 1} \exp \left(\tilde{\alpha} \sum_{\vec{i}} s_{\vec{i}}\left(n_{\vec{i}, \uparrow}-n_{\vec{i}, \downarrow}\right)\right) .
$$

Here, $n_{\vec{i}, \sigma}=c_{\vec{i}, \sigma}^{\dagger} c_{\vec{i}, \sigma}$ where $c_{\vec{i}, \sigma}^{\dagger}\left(c_{\vec{i}, \sigma}\right)$ creates (annihilates) an electron on site $\vec{i}$ with $z$-component of $\operatorname{spin} \sigma, \cosh (\tilde{\alpha})=$ $\exp (\Delta \tau U / 2)$ On an $N$-site lattice, the constant $\tilde{C}=\exp (\Delta \tau U N / 4) / 2^{N}$ and $\Delta \tau$ corresponds to an imaginary time step. As apparent from the above equation, for a fixed set of Hubbard- Stratonovitch (HS) fields, $s_{1} \ldots s_{N}, S U(2)$-spin symmetry is broken. (i.e. the expression is not invariant under the transformation $c_{\vec{j}, \sigma} \rightarrow[\exp (i \phi \vec{e} \vec{\sigma} / 2)]_{\sigma, s^{\prime}} c_{\vec{j}, s^{\prime}}$. Here, $\vec{e}$ is a unit vector and $\vec{\sigma}$ is a vector consisting of the Pauli-spin matrices.) Clearly $S U(2)$ spin symmetry is restored after summation over the HS fields.

Alternatively, one may consider [1]

$$
\exp \left(-\Delta \tau U \sum_{\vec{i}}\left(n_{\vec{i}, \uparrow}-\frac{1}{2}\right)\left(n_{\vec{i}, \downarrow}-\frac{1}{2}\right)\right)=C \sum_{s_{1}, \ldots, s_{N}= \pm 1} \exp \left(i \alpha \sum_{\vec{i}} s_{\vec{i}}\left(n_{\vec{i}, \uparrow}+n_{\vec{i}, \downarrow}-1\right)\right) .
$$

where $\cos (\alpha)=\exp (-\Delta \tau U / 2)$ and $C=\exp (\Delta \tau U N / 4) / 2^{N}$. With this choice of the HS transformation $S U(2)$ spin invariance is retained for any given HS configuration.

The aim of this note, is to address the question: will we obtain a more efficient and/or reliable quantum Monte Carlo algorithm if we enhance the number of symmetries conserved by the HS transformation. We consider the extended Hubbard model:

$$
H=-\frac{t}{2} \sum_{\vec{i}} K_{\vec{i}}-W \sum_{\vec{i}} K_{\vec{i}}^{2}+U \sum_{\vec{i}}\left(n_{\vec{i}, \uparrow}-\frac{1}{2}\right)\left(n_{\vec{i}, \downarrow}-\frac{1}{2}\right)
$$

with the hopping kinetic energy

$$
K_{\vec{i}}=\sum_{\sigma, \vec{\delta}}\left(c_{\vec{i}, \sigma}^{\dagger} c_{\vec{i}+\vec{\delta}, \sigma}+c_{\vec{i}+\vec{\delta}, \sigma}^{\dagger} c_{\vec{i}, \sigma}\right) .
$$

Here $W \geq 0, \vec{\delta}= \pm \vec{a}_{x}, \pm \vec{a}_{y}$ where $\vec{a}_{x}, \vec{a}_{y}$ are the lattice constants. We impose twisted boundary conditions:

$$
c_{\vec{i}+L \vec{a}_{x}, \sigma}=\exp \left(2 \pi i \Phi / \Phi_{0}\right) c_{\vec{i}, \sigma}, \quad c_{\vec{i}+L \vec{a}_{y}, \sigma}=c_{\vec{i}, \sigma},
$$

with $\Phi_{0}=h c / e$ the flux quanta and $L$ the linear length of the square lattice. The boundary conditions given by Eq. (5) account for a magnetic flux threading a torus on which the lattice is wrapped. At half-filling, and constant value of $U / t$ the $W$-term drives the ground state from a antiferromagnetic Mott insulator to a $d_{x^{2}-y^{2}}$ superconductor [2. At $U / t=4$, this quantum transition occurs at $W_{c} / t \sim 0.3$. At finite values of $W<W_{c}$, numerical simulations are consistent with the occurrence of a $d_{x^{2}-y^{2}}$ superconductor upon doping of the Mott insulating state [3]. 
To decompose the $W$-term, we use the approximate relation

$$
e^{\Delta \tau W K_{\vec{i}}^{2}}=\frac{1}{4} \sum_{l=-2,-1,1,2} \gamma(l) \exp \left(\sqrt{\Delta \tau W} \eta(l) K_{\vec{i}}\right)+O\left(\Delta \tau^{4}\right),
$$

where the fields $\eta$ and $\gamma$ take the values:

$$
\begin{array}{r}
\gamma( \pm 1)=1+\sqrt{6} / 3, \quad \gamma( \pm 2)=1-\sqrt{6} / 3 \\
\eta( \pm 1)= \pm \sqrt{2(3-\sqrt{6})}, \quad \eta( \pm 2)= \pm \sqrt{2(3+\sqrt{6})}
\end{array}
$$

Since $K_{\vec{i}}$ is invariant under a rotation in spin space, the above Hubbard-Stratonovitch decomposition conserves $S U(2)$ spin symmetry. Thus, the choice of the HS transformation for the Hubbard term will determine whether the algorithm is $S U(2)$-spin invariant or not.

We have carried out our simulations with the Projector QMC algorithm [4.5]. Within this approach, the ground state expectation value of an observable $O$ is obtained with:

$$
\frac{\left\langle\Psi_{0}|O| \Psi_{0}\right\rangle}{\left\langle\Psi_{0} \mid \Psi_{0}\right\rangle}=\lim _{\Theta \rightarrow \infty} \frac{\left\langle\Psi_{T}\left|e^{-\Theta H} O e^{-\Theta H}\right| \Psi_{T}\right\rangle}{\left\langle\Psi_{T}\left|e^{-2 \Theta H}\right| \Psi_{T}\right\rangle}
$$

The ground state $\left|\Psi_{0}\right\rangle$ is filtered out of a trial wave function, $\left|\Psi_{T}\right\rangle$, provided that $\left\langle\Psi_{0} \mid \Psi_{T}\right\rangle \neq 0$. We choose the trial wave function to be a spin singlet solution of the non interacting Hamiltonian $(U=W=0)$. An explicit construction of such trial wave functions may be found in reference [2].

After Trotter decomposition of the imaginary time propagation and HS transformation of the two-body terms, one obtains:

$$
\frac{\left\langle\Psi_{T}\left|e^{-\Theta H} O e^{-\Theta H}\right| \Psi_{T}\right\rangle}{\left\langle\Psi_{T}\left|e^{-2 \Theta H}\right| \Psi_{T}\right\rangle}=\sum_{x} \operatorname{Pr}(\Theta, x)\langle O\rangle(\Theta, x)+O\left(\Delta \tau^{2}\right),
$$

where $x$ denotes a configuration of HS fields, and $\langle O\rangle(\Theta, x)$, corresponds to the value of the observable $O$ the the HS fields $x$. At half-band filling particle-hole symmetry leads to positive values of $\operatorname{Pr}(\Theta, x)$ which may thus be interpreted as a probability distribution and sampled with Monte Carlo methods. This statement is valid for both choices of the HS transformation of the Hubbard term (1, 2).

We now compare the $S U(2)$ spin invariant algorithm based on Eq. (2) to the $S U(2)$ spin non-invariant algorithm based on Eq. (11). The $S U(2)$ spin-invariant algorithm forces us to work with complex numbers. On the other hand, for many applications real numbers may be used for the $S U(2)$ non-invariant code. For the comparison discussed below, and to keep the CPU time approximately constant, we have carried out twice as many sweeps for the $S U(2)$ non-invariant code than for the $S U(2)$ invariant code. We consider various observables at half-band filling.

a) Magnetization. In the case of the $S U(2)$-invariant algorithm, and the above mentioned choices of the trial wave function one has:

$$
\left\langle c_{\vec{i}, \uparrow}^{\dagger} c_{\vec{j}, \uparrow}\right\rangle(\Theta, x)=\left\langle c_{\vec{i}, \downarrow}^{\dagger} c_{\vec{j}, \downarrow}\right\rangle(\Theta, x)
$$

Thus, the total magnetization, $m_{z}(\vec{q})=\sum_{\vec{j}} e^{i \vec{q} \vec{j}}\left(n_{i, \uparrow}-n_{i, \downarrow}\right)$ is identical to zero for all values of the HS fields:

$$
\left\langle m_{z}(\vec{q})\right\rangle(\Theta, x) \equiv 0 .
$$

On the other hand, the $S U(2)$ non-invariant algorithm, equation (10) is not valid, and one obtains zero magnetization, only after summation over the HS fields. At $\vec{q}=(\pi, \pi) \equiv \vec{Q}, L=6,\langle n\rangle=1, U / t=4$ and $W / t=0$ one obtains after $2 \times 10^{5}$ sweeps, $\left\langle m_{z}(\vec{Q})\right\rangle=-0.16 \pm 0.31$. Thus for this trivial case, the advantage of the $S U(2)$ invariant algorithm over the $S U(2)$ non-invariant algorithm is infinite. 


$$
L=6, W / t=0.0, U / t=4.0,\langle n\rangle=1, T=0
$$

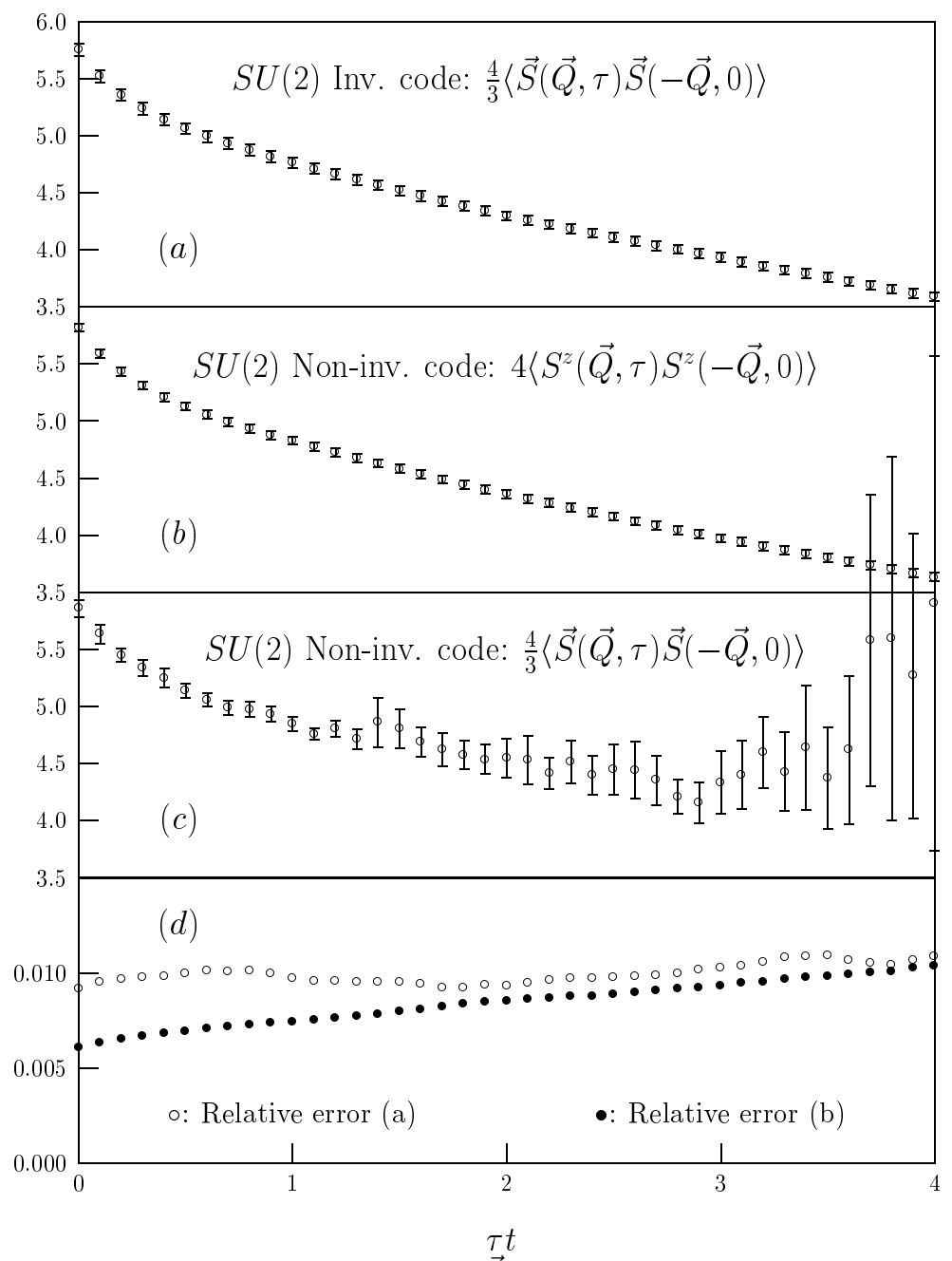

FIG. 1. Imaginary time displaced spin-spin correlations, at $\vec{Q}=(\pi, \pi)$. The $S U(2)$ invariant code is based on the HS transformation of Eq. (2) and $S U(2)$ non-invariant on Eq. (1). To keep the CPU time approximately constant between the two simulations, we have carried out twice as many sweeps for the $S U(2)$ non-invariant code as for the $S U(2)$ invariant code. Here, we have used periodic boundary conditions, $\Phi=0$ in Eqn. (五).

b) Imaginary time displaced spin-spin correlations. Here we consider the quantity: $S^{\alpha}(\vec{q}, \tau) S^{\alpha}(-\vec{q}, 0)$ where $S^{\alpha}(\vec{q}, \tau)=\sum_{\vec{j}} e^{i \vec{q} \vec{j}} e^{\tau H} S_{j}^{\alpha} e^{-\tau H}, S_{j}^{\alpha}$ being the $\alpha$-component of the spin operator on site $\vec{j}$. The numerically stable computation of imaginary time displaced correlation functions within the Projector QMC algorithm is described in Ref. [6]. In the $\mathrm{SU}(2)$-invariant algorithm one obtains with the above mentioned trial wave function:

$$
\left\langle S^{\alpha}(\vec{q}, \tau) S^{\alpha}(-\vec{q}, 0)\right\rangle(\Theta, x) \equiv\left\langle S^{\gamma}(\vec{q}, \tau) S^{\gamma}(-\vec{q}, 0)\right\rangle(\Theta, x) .
$$

Here, $\alpha, \gamma$ run over the three components of the spin. In the case of the $\mathrm{SU}(2)$ non-invariant the above equation is valid only after summation over the HS fields, $x$. Fig. 1 plots the spin-spin correlations for $\langle n\rangle=1, U / t=4$ and $W / t=0$ on a $6 \times 6$ lattice. The half-filled Hubbard model is expected to show long-range antiferromagnetic order in the thermodynamic limit. Thus, $\frac{1}{N}\langle\vec{S}(\vec{Q}, \tau) \vec{S}(-\vec{Q}, 0)\rangle$ is should saturate to a finite value in the large $L$ and $\tau$ limits. Here, $L$ is the linear size of the square lattice and $N$ the number of sites. On a finite size lattice, a spin gap is expected, thus leading to an exponential decay in $\tau$ of the considered quantity. If one compares the quantity $\langle\vec{S}(\vec{Q}, \tau) \vec{S}(-\vec{Q}, 0)\rangle$ for both codes, (Fig. 1a and Fig. 1c) it is clear that the $S U(2)$ invariant code does much better. The large fluctuations in the case of the $S U(2)$ non-invariant code may be traced back to the $x$ and $y$-components of the spin-spin correlation function. In fact, considering only the $z$-component of the correlation function (Fig. 1b) yields good results. The HS transformation of equation (11) conserves the $z$-component of the total spin, but not the other components. We now consider the $z$-component of the spin-spin correlations and compare both algorithms. We have plotted in Fig. 1d the relative errors for both codes. 


$$
L=8, W / t=0.35, U / t=2.0,\langle n\rangle=1, T=0, \Phi=\Phi_{0} / 2
$$

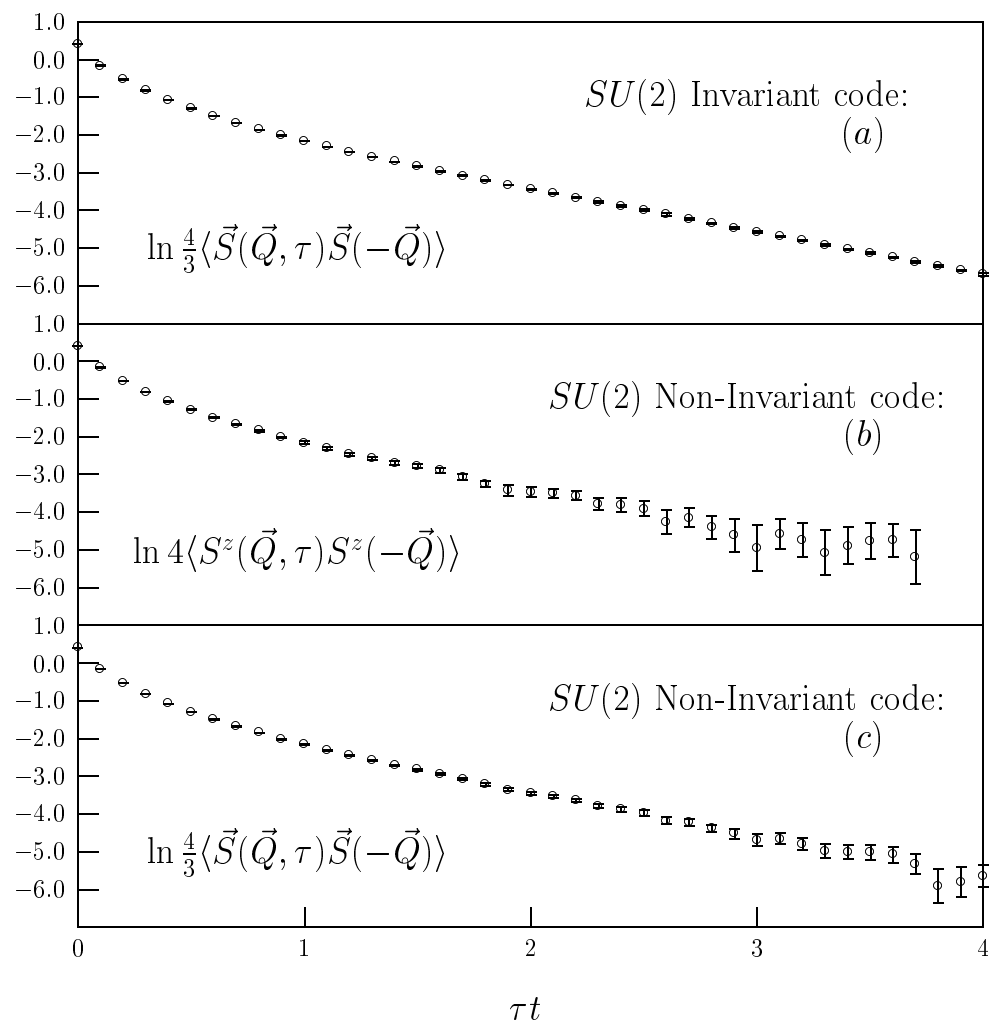

FIG. 2. Same as Fig. 11 for the parameter set: $W / t=0.35, U / t=2$ and at half-band filling. Here we use antiperiodic boundary conditions set $\Phi=\Phi_{0} / 2$. It is clear that the $S U(2)$-invariant code does substantially better at large values of $\tau$.

Overall, the $\mathrm{SU}(2)$ spin-invariant code produces larger errors within the considered $\tau$-range. However, for the $S U(2)$-invariant code the relative error, is to a first approximation independent of $\tau$. In contrast, the relative error for the $S U(2)$ non-invariant code grows as a function of $\tau$. At large values of $\tau$ we expect the the $S U(2)$ invariant code to be more efficient. To confirm this statement, we consider the parameter set: $\langle n\rangle=1, U / t=2, W / t=0.35$, $\Phi=\Phi_{0} / 2$ on a $8 \times 8$ lattice. The data is shown in Fig.2. It is clear that the $S U(2)$-invariant code does much better at large values of $\tau t$. In Fig. 2, the $z$-component of the spin-spin correlations ( Fig. $2 \mathrm{~b}$ ) shows larger fluctuations than $\langle\vec{S}(\vec{Q}, \tau) \vec{S}(-\vec{Q}, 0)\rangle$ ( Fig. 2c). Exactly the opposite is seen in Figs. 1b and 1c.

\begin{tabular}{|c|c|c|c|c|}
\hline \hline$\tau t$ & $\begin{array}{c}S U(2) \text { invariant code } \\
\left\langle\Pi^{z}(\tau) \Pi^{z, \dagger}(0)\right\rangle\end{array}$ & $\begin{array}{c}S U(2) \text { non-invariant code } \\
\left\langle\Pi^{x}(\tau) \Pi^{x, \dagger}(0)\right\rangle\end{array}$ & $\begin{array}{c}S U(2) \text { non-invariant code } \\
\left\langle\Pi^{z}(\tau) \Pi^{z, \dagger}(0)\right\rangle\end{array}$ \\
\hline 0 & $0.15055 \pm 0.00080$ & $0.15097 \pm 0.00073$ & $0.13837 \pm 0.01506$ \\
0.2 & $0.05902 \pm 0.00058$ & $0.05920 \pm 0.00051$ & $0.05845 \pm 0.00387$ \\
0.4 & $0.02915 \pm 0.00055$ & $0.02941 \pm 0.00040$ & $0.02846 \pm 0.00215$ \\
0.6 & $0.01474 \pm 0.00046$ & $0.01547 \pm 0.00032$ & $0.01373 \pm 0.00254$ \\
0.8 & $0.00728 \pm 0.00046$ & $0.00859 \pm 0.00039$ & $0.00841 \pm 0.00105$ \\
1 & $0.00397 \pm 0.00038$ & $0.00480 \pm 0.00053$ & $0.00394 \pm 0.00104$ \\
1.2 & $0.00240 \pm 0.00033$ & $0.00251 \pm 0.00033$ & $0.00086 \pm 0.00080$ \\
\hline \hline
\end{tabular}

TABLE I. П-mode correlation functions at half-filling for $U / t=4, W / t=0.35$ on a $6 \times 6$ lattice. Data in the last two columns were obtained with the SU(2) non-invariant code. Within this algorithm, the $x$ and $y$ components of the $\Pi$-mode correlation function are identical. For the $S U(2)$ spin-invariant algorithm, the results are independent on the considered components of the correlation function. To keep the CPU time approximately constant between the two simulations, we have carried out twice as many sweeps for the $S U(2)$ non-invariant code as for the $S U(2)$ invariant code. 
c) $\Pi$-modes. The $\Pi$ modes introduced in the $S O(5)$ theory of the unification of antiferromagnetism and supercon-

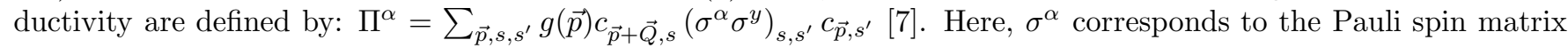
and for the case of $d$-wave superconductivity, we consider $g(\vec{p})=\cos \left(p_{x}\right)-\cos \left(p_{y}\right)$. The $S U(2)$-spin invariant code satisfies

$$
\left\langle\Pi^{\alpha}(\tau) \Pi^{\alpha, \dagger}(0)\right\rangle(\Theta, x) \equiv\left\langle\Pi^{\gamma}(\tau) \Pi^{\gamma, \dagger}(0)\right\rangle(\Theta, x) .
$$

In the case of the $S U(2)$ non-invariant code, the above equation is valid for all values of $\gamma$ and $\alpha$ only after summation over the HS fields $x$. In the table, imaginary time $\Pi$ correlations are considered for both algorithms. For the $S U(2)$ non-invariant code, substantial fluctuations in $\left\langle\Pi^{z}(\tau) \Pi^{z, \dagger}(0)\right\rangle$ are observed at large values of $\tau$. On the other hand, the error-bars in $\left\langle\Pi^{x}(\tau) \Pi^{x, \dagger}(0)\right\rangle$ are smaller. We again attribute the large fluctuations in the $z$ component of the $\Pi$ mode correlations to fact that $\Pi^{z}$ does not commute with the $z$-component of the total spin. In contrast, $\Pi^{x}$ does commute with the $z$-component of the total spin. The $S U(2)$-invariant code shows good convergence, and the results agree with those obtained for the x-component of the П-mode within the $S U(2)$ non-invariant algorithm.

d) Single particle Green functions. We have not found any significant improvements in the fluctuations of the imaginary time single particle Green functions between the two algorithms. To be more precise, the fluctuation of $\frac{1}{N} \sum_{\sigma, \vec{i}}\left\langle c_{\vec{i}, \sigma}(\tau) c_{\vec{i}, \sigma}^{\dagger}\right\rangle$ are to a first approximation independent of the choice of HS transformation. Thus for both considered codes, the error-bars scale as $C / \sqrt{N_{\text {sweeps }}}$ where $C$ is a constant independent on the choice of the algorithm, and $N_{\text {sweeps }}$ denotes the number of sweeps carried out.

In conclusion, we have considered an alternative HS transformation for Hubbard type models which conserves $S U(2)$ spin symmetry. This algorithm requires the use of complex numbers and does not introduce a sign problem at half-band filling. In the case of $S U(2)$ non-invariant algorithms based on Eq. (11), very large fluctuations can occur in the calculation of imaginary time displaced quantities of the form $\left\langle A(\tau) A^{\dagger}\right\rangle$ when $A$ does not commute with the $z$-component of the total spin. In the $S U(2)$ invariant formulation, this pathology does not occur. We have compared the two algorithms in the worst case scenario where real numbers can be used for the $S U(2)$ non-invariant algorithm. By keeping the CPU time constant for both codes we have shown that the long imaginary time behavior of the spin-spin correlations are obtained more efficiently with the $S U(2)$ invariant code than with the $S U(2)$ non-invariant code. Thus, the $S U(2)$ invariant code is more efficient for the measure of spin gaps which may be extracted from the long imaginary time decay of the spin-spin correlations. More generally, it is an efficient code for the study of spin dynamics. The fluctuations of other quantities such as single particle green function, were invariant under the choice of the HS transformation.

\section{ACKNOWLEDGMENTS}

M. Muramatsu is thanked for many instructive conversations. The computations were carried out on the T3E of the HLRS,Stuttgart, as well as on the T90 and T3E of the HLRZ, Jülich.

[1] J.E.Hirsch, Phys. Rev. B 284059 (1983).

[2] F.F. Assaad, M. Imada and D.J. Scalapino, Phys. Rev. Lett. 77, 4592, (1996). Phys. Rev. B 56, 15001, (1997).

[3] F.F. Assaad and M. Imada Phys. Rev. B. 58, (1998).

[4] G. Sugiyama and S.E. Koonin, Annals of Phys.168 1, (1986).

[5] S. Sorella, S. Baroni, R. Car, And M. Parrinello, Europhys. Lett. 8, 663, (1989). S. Sorella, E. Tosatti, S. Baroni, R. Car, and M. Parinello, Int. J. Mod. Phys. B1, 993, (1989).

[6] F.F. Assaad and M. Imada, J. Phys. Soc. Jpn. 65,189, (1996).

[7] S.C. Zhang, Science, 275, 1089, (1997). 